\title{
Cognitive decline tracks motor progression and not disease duration in Parkinson patients
}

\author{
BD Riggeal' \\ GP Crucian' \\ P Seignourel $^{2}$ \\ CE Jacobson IV' \\ MS Okun' \\ RL Rodriguez' \\ Hubert H Fernandez' \\ 'Department of Neurology; \\ ${ }^{2}$ Department of Community Health \\ and Psychology, University of Florida, \\ Gainesville, FL, USA
}

\begin{abstract}
We performed an analysis of prospectively-acquired cross sectional data on 106 Parkinson disease (PD) patients who underwent comprehensive neuropsychological testing and the Unified Parkinson Disease Rating Scale (UPDRS) motor scale. A significant correlation between the UPDRS motor and neuropsychological tests in all cognitive domains except for general intelligence and visuo-spatial function was seen. In this study, cognitive decline within this PD cohort correlated with motor impairment but not disease duration. Our findings suggest that overall cognitive impairment (except visuospatial dysfunction) may track motor progression in PD more than duration of disease. Longitudinal studies are needed to confirm our results.
\end{abstract}

Keywords: Parkinson, dementia, cognition, visual-spatial dysfunction

\section{Introduction}

It has been previously thought that dementia occurred in $20 \%-30 \%$ of Parkinson disease (PD) patients, usually at the later stage of their illness. However, recent long term epidemiological studies reported the prevalence rate of dementia in PD closer to $50 \%$ (Hely et al 2005). Others have reported that some form of cognitive decline affects nearly $80 \%$ of PD patients (Aarsland et al 2003). The relative risk for the development of dementia in PD has been reported as being between 1.7 to 5.9 (Marder et al 1995; Aarsland et al 2001; Hobson et al 2004).

We hypothesize that cognitive dysfunction in PD is progressive, actually begins early in the course, affects most patients, and tracks their motor impairment. Moreover, because of the variability of PD severity among affected individuals, we further hypothesize that cognitive measures are less likely to be correlated with disease duration: a patient with "mild disease" may have had PD for several years with minimal motor and, therefore, cognitive impairment; while a patient with an aggressive disease course may be significantly impaired (physically and cognitively) sooner.

\section{Methods}

All patients diagnosed with "probable" PD at a single Movement Disorders Center were offered to undergo a comprehensive neuropsychological testing, regardless of their cognitive status. The results were correlated to the total motor score of the Unified Parkinson Disease Rating Scale (UPDRS).

The diagnosis of "probable" PD, made by either of 3 fellowship-trained Movement Disorders Specialists (HHF, MSO, RLR), was determined by: (1) the presence of two out of three motor features of PD (tremor, rigidity, and bradykinesia), (2) and the absence of any features that would suggest other forms of Parkinsonism including lack of a substantial response to levadopa therapy. For all medicated patients, the UPDRS was performed in the "off" state (ie, at least 12 hours off all PD medications). There were no patients with deep brain stimulators included in our study.
Correspondence: Hubert H Fernandez PO Box 100236, Gainesville, FL 326I0, USA

Tel + I 3522735575

Fax +I 3522735550

Email fernandez@neurology.ufl.edu 
The neuropsychological battery (performed in the "on" state, if medicated) included: the Mini-Mental Status Exam (MMSE); Dementia Rating Scale (DRS); and tests on the following domains: general intelligence (Wechsler Adult Intelligence Scale 3); academic performance (Wide Range Achievement Test-3 [WRAT-3]); attention and concentration (Digit Span); memory (Hopkins Verbal Learning Test [HVLT] and Weschler Memory Scale-III); language (Boston Naming, Controlled Oral Word Association Test); visualspatial skills (Judgment of Line Orientation [JOLO], Benton Facial Recognition Test); and executive function (Stroop, Trail Making Test).

Demographic data including age, disease duration and education were also obtained.

The correlation of the UPDRS motor scores to neuropsychological performance (both raw scores and corrected scores for age and education) was then determined. Because some of the variables were found to deviate substantially from normality, nonparametric correlations (Spearman's) were computed to examine the relationship between all variables. Since our study is exploratory, we did not apply a correction for multiple comparisons to determine significance in our primary analyses. However, we indicated which correlations remained significant after a Bonferroni correction as a secondary analysis. To make sure that observed relationships were not solely due to the effects of intervening variables, multiple regressions were conducted to examine the unique relationship between motor and cognitive functioning after controlling for demographic characteristics and disease duration. All analyses were conducted with SPSS 13.0. The collection and utilization of data in this study is IRB approved.

\section{Results}

One hundred and six PD patients, 30 females and 76 males, were included in this study. Two participants were excluded based on very poor cognitive functioning (MMSE of 7 and 14). The participants had an average: age of 67.1-years (SD: 10.52); education of 14.75-years (SD: 3.38); disease duration of 110.41-months (SD: 74.43); MMSE score of 27.58 (range: 18-30, SD: 2.45); and UPDRS motor "off" score of 38.40 (SD: 13.50). Only two patients were medication-naive in this cohort.

The tests that significantly correlated with the UPDRS motor score were: MMSE, DRS, Digit Span, HVLT, WMS-III, Boston Naming Test, Letter Fluency, Animal Fluency, Stroop Color Naming and Trail Making Test B, representing at least one component in the neuropsychological domains of attention and concentration, memory, language, and executive function (see Table 1). However, some tests under these cognitive domains did not correlate with the UPDRS motor score, including the Stroop word reading, Stroop color-word naming, and Trail Making Test A. Tests of general intelligence (WAIS-III), academic performance (WRAT-3), and visuospatial skills (JOLO and face recognition) showed no significant relationship with the UPDRS motor score.

Age, gender, and education were not significantly associated with the UPDRS motor score. Disease duration, however, was significantly and positively correlated with UPDRS motor score (see Table 1). A multiple regression including UPDRS motor score and disease duration as independent predictors yielded UPDRS motor score as the sole unique predictor of MMSE $\left(\mathrm{R}^{2}=0.09, \mathrm{~F}(2,76)=3.69\right.$, $\mathrm{p}<0.05, \beta($ UPDRS $)=-0.33, \mathrm{t}(76)=-2.71, \mathrm{p}<0.01)$. Even as a single predictor, disease duration was not significantly correlated with MMSE $(r=-0.01, p>0.1$, or DRS, $r=-0.13, p>0.1)$.

\section{Discussion}

Our results showed a significant correlation between the degree of motor impairment and most of the neuropsychological tests in all cognitive domains except for general intelligence and visuospatial function. This implies that the cognitive decline seen with PD is near 'global' rather than primarily being a 'dysexecutive syndrome' (Litvan et al 1991). Moreover, although our study is cross-sectional, it suggests that cognitive decline tracks motor progression in PD although longitudinal studies are needed to confirm this observation.

Although studies have found significant visuospatial disturbances associated with PD (Huber et al 1989; Stern et al 1993), ours did not show a correlation between motor score and visuospatial abilities. This does not mean that visuospatial dysfunction did not occur in our cohort. This simply suggests that visuospatial dysfunction did not track motor progression. Possible explanations include: stronger influence of external factors (eg, testing environment, medications) in visuospatial tests compared to measures of other cognitive domains; insensitivity of visuospatial tests used in this study; or, a difference or divergence in the etiopathogenesis of visual-spatial dysfunction in PD compared with the motor and other cognitive dysfunction. Also, it has been thought by some that visuospatial disturbances experienced by PD patients may be secondary to or at least influenced by executive dysfunction and inattention (Ogden 
Table I Nonparametric correlations between UPDRS scores and neuropsychological performance

\begin{tabular}{|c|c|c|c|c|}
\hline & \multicolumn{4}{|c|}{ UPDRS total score } \\
\hline & \multicolumn{2}{|c|}{ Raw scores } & \multicolumn{2}{|c|}{ Corrected scores ${ }^{a}$} \\
\hline & $\mathrm{R}$ & $\mathrm{p}$-value ${ }^{\mathrm{b}}$ & $\mathrm{R}$ & $\mathrm{p}$-value ${ }^{\mathrm{b}}$ \\
\hline MMSE $(\mathrm{N}=106)$ & -0.32 & $<0.001 *$ & - & - \\
\hline Dementia Rating Scale $(\mathrm{N}=106)$ & -0.28 & $<0.01$ & -0.28 & $<0.01$ \\
\hline WAIS-III Information (N = 102) & -0.14 & 0.15 & -0.12 & 0.23 \\
\hline WAIS-III Vocabulary ( $\mathrm{N}=98)$ & -0.13 & 0.22 & -0.13 & 0.21 \\
\hline Digit Span Total $(\mathrm{N}=104)$ & -0.19 & $<0.05$ & -0.25 & $<0.01$ \\
\hline Digit Span Forward (N = 104) & -0.16 & 0.11 & -0.26 & $<0.01$ \\
\hline Digit Span Backward $(\mathrm{N}=104)$ & -0.06 & 0.58 & -0.11 & 0.26 \\
\hline HVLT Ist trial $(\mathrm{N}=104)$ & -0.27 & $<0.01$ & -0.30 & $<0.01 *$ \\
\hline HVLT Total $(\mathrm{N}=104)$ & -0.23 & $<0.05$ & -0.27 & $<0.01$ \\
\hline HVLT 20-minute Delay $(\mathrm{N}=104)$ & -0.22 & $<0.05$ & -0.26 & $<0.01$ \\
\hline HVLT Recognition ( $\mathrm{N}=104$ ) & -0.26 & $<0.01$ & -0.30 & $<0.01$ \\
\hline WMS-III Logical Memory I $(N=103)$ & -0.29 & $<0.01$ & -0.32 & $<0.00 I^{*}$ \\
\hline WMS-III Logical Memory II $(\mathrm{N}=103)$ & -0.28 & $<0.01$ & -0.31 & $<0.01 *$ \\
\hline WRAT-3 Total $(\mathrm{N}=98)$ & -0.06 & 0.58 & -0.10 & 0.34 \\
\hline Boston Naming Test $(\mathrm{N}=106)$ & -0.32 & $<0.00 I^{*}$ & -0.31 & $<0.01 *$ \\
\hline Letter Fluency ( $\mathrm{N}=104)$ & -0.44 & $<0.00 I^{*}$ & -0.42 & $<0.00 I^{*}$ \\
\hline Animal Fluency $(\mathrm{N}=104)$ & -0.28 & $<0.01$ & -0.33 & $<0.00 I^{*}$ \\
\hline Judgment Of Line Orientation ( $N=95)$ & -0.06 & 0.55 & -0.15 & 0.16 \\
\hline Face Recognition Test $(\mathrm{N}=98)$ & -0.15 & 0.15 & -0.19 & 0.06 \\
\hline Stroop Word Reading $(\mathrm{N}=100)$ & -0.10 & 0.34 & -0.11 & 0.27 \\
\hline Stroop Color Naming $(\mathrm{N}=93)$ & -0.21 & $<0.05$ & -0.23 & $<0.05$ \\
\hline Stroop Color-Word Naming $(\mathrm{N}=105)$ & -0.17 & 0.09 & -0.19 & 0.06 \\
\hline Trail Making Test A (N = 102) & 0.11 & 0.29 & -0.16 & 0.12 \\
\hline Trail Making Test $\mathrm{B}(\mathrm{N}=98)$ & 0.21 & $<0.05$ & -0.12 & 0.28 \\
\hline Disease Duration & 0.46 & $<0.001 *$ & - & - \\
\hline
\end{tabular}

Notes: ${ }^{\text {TThis }}$ column refers to correlations between the UPDRS and age-corrected (WAIS-III Information and Vocabulary, Digit Span, HVLT,WMS-III Logical Memory, WRAT-3 and Stroop) or age-and-education corrected scores (Dementia Rating Scale, Boston Naming Test, Letter and Animal Fluency, Judgment of Line Orientation, Face

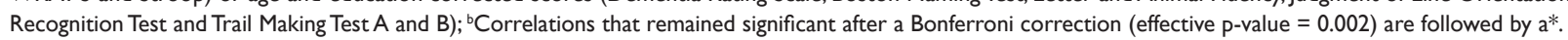

et al 1990; Bondi et al 1993). The visuospatial tests used in our study were not associated with the motor UPDRS scores, but representative tests of both executive function and attention were.

The correlation between the MMSE, representing the overall cognitive status, and the UPDRS motor score remained significant even after controlling for disease duration. On the contrary, no correlation was found between disease duration and MMSE or DRS.

Several weaknesses in the study should be noted. The cohort consisted of patients treated at a single PD Center. Not all subjects agreed to be tested. We did not take note of the total number of subjects offered to undergo testing. It is therefore possible that patients with signs of dementia more likely agreed to undergo the neuropsychiatric evaluation, contributing to a population bias. Also, patients with severe cognitive deficits who were unable to complete the comprehensive neuropsychological examination $(\mathrm{N}=2)$ were excluded. Nonetheless, we believe that this cohort represented a good spectrum of PD. The small number of excluded patients due to severe dementia (2/108) was not likely to influence overall results. Although our 'convenience sample' could be subject to population bias, the range in age (41-89), MMSE scores (18-30), disease duration (12 to 318-months), UPDRS scores (8-81), represented a wide PD spectrum.

All neuropsychological examination was performed in the "on" state. Thus, it is less likely that motor fluctuations significantly contributed to their performance during cognitive testing. The motor UPDRS was obtained in the "off" state to ensure uniformity, assess the true/baseline motor deficits, without medication effect or the variability of its optimization. Only 2 patients in our cohort were medication-naïve. Finally, this is a cross-sectional study so our data should be interpreted with caution.

In summary, the results of this study indicate that impairment in most cognitive domains correlate with motor symptoms in PD. Cognitive impairment may start early in the disease process but may be very mild and often undetected. While most clinicians are able to appreciate mild tremor or rigidity in one limb thereby easily "defining" the onset of motor dysfunction, the first sign of cognitive impairment is 
more difficult to identify. Longitudinal studies are needed to confirm our results as our cross-sectional study can indicate correlation of cognitive impairment and motor symptoms, but is insufficient to detect true parallel progression of cognitive and motor impairment. If proven correct, then (1) better and practical cognitive tests sensitive to PD that mark the onset and track the progression of cognitive dysfunction are needed; and, (2) cognition enhancing or modifying (pharmacologic and non-pharmacologic) interventions, as they become available, should be considered early in the course of PD.

Finally, this study does not support previous reports regarding visuospatial impairment in PD. Controlled longitudinal studies with the use of more sensitive measures may be needed to define the etiopathogenesis of visuospatial dysfunction in PD.

\section{Disclosures}

The authors report no conflict of interest. This study was partly funded by a T35 Training Grant from the NIH.

\section{References}

Aarsland D, Andersen K, Larsen JP, et al. 2001. Risk of dementia in Parkinson's disease: a community-based, prospective study. Neurology, 56:730-6.

Aarsland D, Anderson K, Larsen JP, et al. 2003. Prevalence and Characteristics of Dementia in Parkinson's Disease: an 8-year Prospective Study. Arch Neurol, 60:387-92.
Bondi MW, Kaszniak AW, Bayles KA, et al. 1993. Contribution of frontal system dysfunction to memory and perceptual abilities in Parkinson's disease. Neuropsychology, 7:89-102.

Hely MA, Morris JG, Reid WG, et al. 2005. Sydney Multicenter Study of Parkinson's disease: non-L-dopa-responsive problems dominate at 15-years. Mov Disord, 20:190-9.

Hobson P, Meara J. 2004. Risk and Incidence of Dementia in a Cohort of Older Subjects With Parkinson's Disease in the United Kingdom. Mov Disord, 19:1043-9.

Huber SJ, Shuttleworth EC, Freidenberg DL. 1989. Neuropsychological differences between the dementias of Alzheimer's and Parkinson's diseases. Arch Neurol, 46:1287-91.

Litvan I, Mohr E, Williams J, et al. 1991. Differential memory and executive functions in demented patients with Parkinson's and Alzheimer's disease. J Neurol Neurosurg Psychiatry, 54:25-29.

Marder K, Tang MX, Cote L, et al. 1995. The frequency and associated risk factors for dementia in patients with Parkinson's disease. Arch Neurol, 52:695-701.

Ogden JA, Growdon JH, Corkin S. 1990. Deficits on visuospatial tests involving forward planning in high-functioning Parkinsonians. Neuropsychiatr Neuropsychol Behav Neurol, 3:125-39.

Stern Y, Richards M, Sano M, et al. 1993. Comparison of cognitive changes in patients with Alzheimer's and Parkinson's disease. Arch Neurol, 50:1040-5. 